\title{
Stationary Points-I: One-Dimensional p-Fuzzy Dynamical Systems
}

\author{
Joao de Deus M. Silva, ${ }^{1}$ Jefferson Leite, ${ }^{2}$ Rodney C. Bassanezi, ${ }^{3}$ and Moiseis S. Cecconello ${ }^{4}$ \\ ${ }^{1}$ CCET UFMA, 65085-558 Sao Luis, MA, Brazil \\ ${ }^{2}$ DEMAT CCN, UFPI, 64063040 Teresina, PI, Brazil \\ ${ }^{3}$ CMCC UFABC, 09210-170 Santo Andre, SP, Brazil \\ ${ }^{4}$ DMAT ICET UFMT, 78075-202 Cuiaba, MT, Brazil
}

Correspondence should be addressed to Jefferson Leite; jleite@ufpi.edu.br

Received 13 April 2013; Accepted 1 August 2013

Academic Editor: Toly Chen

Copyright (C) 2013 Joao de Deus M. Silva et al. This is an open access article distributed under the Creative Commons Attribution License, which permits unrestricted use, distribution, and reproduction in any medium, provided the original work is properly cited.

p-Fuzzy dynamical systems are variational systems whose dynamic is obtained by means of a Mamdani type fuzzy rule-based system. In this paper, we will show the 1-dimensional p-fuzzy dynamical systems and will present theorems that establish conditions of existence and uniqueness of stationary points. Besides the obtained analytical results, we will present examples that illustrate and confirm the obtained mathematical results.

\section{Introduction}

Variational equations or deterministic dynamical systems (difference and differential equations) constitute a powerful tool for modeling when the state variables depend on variations throughout time. The efficiency of a deterministic model depends on knowledge of the relationships between variables and their variations. In addition, in many situations such relations are only partially known; therefore, the modeling with deterministic variational systems, or even with stochastic ones, may not be adequate. In addition, fuzzy systems derived from deterministic models, which have subjectivity regarding some parameters, are not appropriate when we have only incomplete information of the phenomenon being analyzed. Thus, the use of a rule-based system can be adopted as an alternative for modeling partially known phenomena or those carried with imprecision.

Fuzzy rule-based systems have been used with success in some areas as control, decision taking, recognition systems, and so forth. This success is due to its simplicity and interrelation with humans way of reasoning. Fuzzy rulebased systems are conceptually simple [1]. Such systems are basically threefold: an input (fuzzifier), an inference mechanism composed of a base of fuzzy rules together with an inference method, and, finally, an output (defuzzifier) stage (see Figure 1).

There are two main types of fuzzy rule-based systems, the Mamdani fuzzy systems and the Takagi-Sugeno fuzzy systems [2]. The main characteristic of the Mamdani type systems is that both the antecedent and consequent are expressed by linguistic terms, while in the Takagi-Sugeno type systems only the antecedent is expressed by linguistic terms and the consequent is expressed by functions.

The Takagi-Sugeno fuzzy systems are more restrictive than the Mamdani fuzzy systems because they require an $a$ priori function in the output. However, due to the existence of theoretical methods for Takagi-Sugeno fuzzy systems stability analysis [3-8], the latter has become more used. On the other hand, Mamdani type systems are used as a "black box" and are still criticized because they lack a study on its stability $[9,10]$.

Fuzzy variational equations have been used in different methods. Some attempts to contemplate subjectivity original from aleatory processes such as Hukuhara's derivative, differential inclusions, and Zadeh's extension [11] have been already proposed. In these methods, the adopted process for studying the variational systems is always derived from deterministic classical systems. 


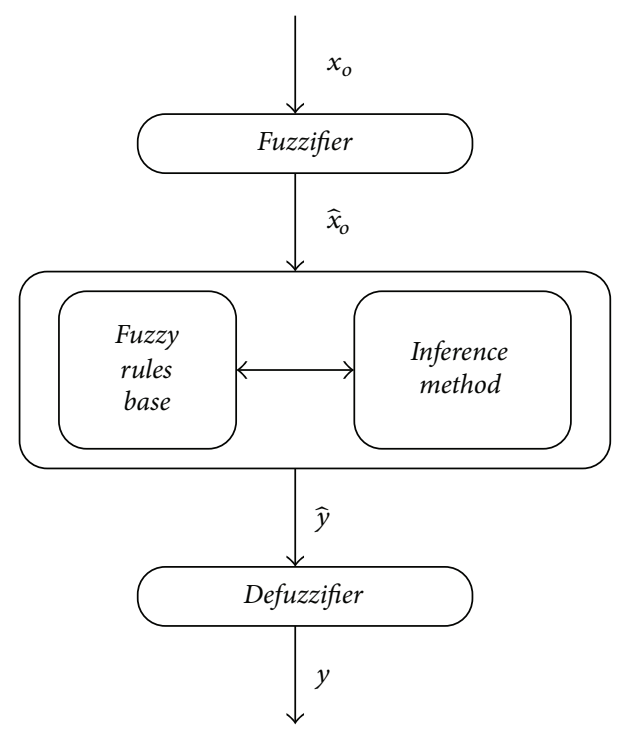

FIgURE 1: Architecture of a fuzzy rule-based system.

In this paper, we will present the p-fuzzy systems whose dynamics is not based in formal concepts of variations originated from derivative or explicit differences or differential inclusions. In the p-fuzzy dynamical systems the dynamic (iterative process) is obtained by means of a Mamdani's fuzzy rule-based system. The main advantage of this method with respect to the other ones is the simplicity of the involved mathematics, just because the interactive method is supplied by the Mamdani controller.

Formally, a p-fuzzy system in $\mathbb{R}^{n}$ is a discrete dynamic system:

$$
\begin{gathered}
x_{k+1}=F\left(x_{k}\right), \\
x_{o} \text { given and } x_{k} \in \mathbb{R}^{n},
\end{gathered}
$$

where the $F$ function is given by $F\left(x_{k}\right)=x_{k}+\Delta\left(x_{k}\right)$ and $\Delta\left(x_{k}\right) \in \mathbb{R}^{n}$ is obtained by means of a fuzzy rule-based system; that is, $\Delta\left(x_{i}\right)$ is the defuzzification value of the rulebased system. The architecture of a p-fuzzy system can be visualized in Figure 2.

The name p-fuzzy dynamical systems or purely fuzzy was chosen to differentiate it from other fuzzy systems given by variational equations.

In this paper, we will focus on the one-dimensional pfuzzy systems, which are always associated with a Mamdani fuzzy system, where the defuzzification method is the centroid. We have chosen this method because it is widely used and more general to deal with weight mean of linguistic variables [12, pages 242,243$]$.

Analogous to the inhibited variational models in which one has stationary solutions, our objective is to present results that establish the necessary and sufficient conditions for the existence of a stationary point.

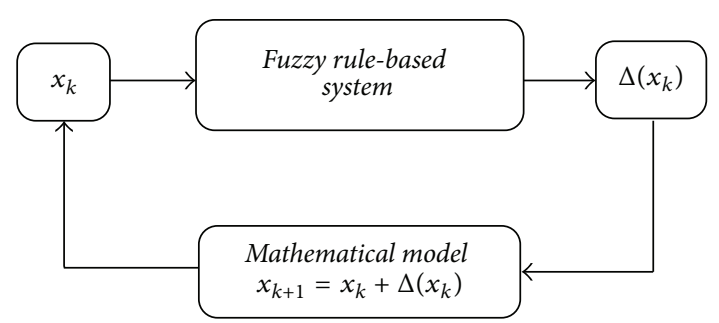

FIGURE 2: Architecture of a p-fuzzy system.

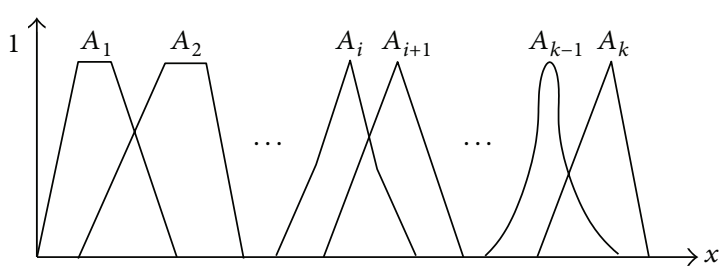

FIGURE 3: Family of successive fuzzy subsets.

\section{Preliminaries}

In this section, we introduce the main concepts for the development of the work presented in this paper.

\subsection{Definitions}

Definition 1 (support). Let $A$ be a fuzzy subset of $X$; the support of $A$, denoted $\operatorname{supp}(A)$, is the crisp subset of $X$ whose elements all have nonzero membership grades in $A$; that is,

$$
\operatorname{supp}(A)=\{x \in X ; A(x)>0\} .
$$

Definition 2 ( $\alpha$-cut). An $\alpha$-level set of a fuzzy subset $A$ of $X$ is a crisp set denoted by $[A]^{\alpha}$ and defined by

(i) $[A]^{0}=\overline{\operatorname{supp}(A)},(\alpha=0)$,

(ii) $[A]^{\alpha}=\{x \in X ; A(x) \geq \alpha\}$, if $\alpha \in(0,1]$.

Definition 3 (fuzzy number). A fuzzy number $A$ is a fuzzy set $A \subset \mathbb{R}$ satisfying the following conditions:

(i) $[A]^{\alpha} \neq \varnothing, \forall \alpha \in[0,1]$,

(ii) $[A]^{\alpha}$ is a closed interval, $\forall \alpha \in[0,1]$,

(iii) the $\operatorname{supp}(A)$ is bounded.

Definition 4. Consider a one-dimensional p-fuzzy system given by (1).

Consider that $x^{*}$ is a stationary point of (1) if

$$
F\left(x^{*}\right)=x^{*}+\Delta\left(x^{*}\right)=x^{*} \Longleftrightarrow \Delta\left(x^{*}\right)=0 .
$$

Definition 5. Let $\left\{A_{i}\right\}_{1 \leq i \leq k}$ be any finite family of normal fuzzy subsets associated with the fuzzy variable $x$. Assume that $\left\{A_{i}\right\}_{1 \leq i \leq k}$ is a family of successive fuzzy subsets (Figure 3 ) if,

(i) $\operatorname{supp}\left(A_{i}\right) \cap \operatorname{supp}\left(A_{i+1}\right) \neq \varnothing$, for each $1 \leq i<k$; 


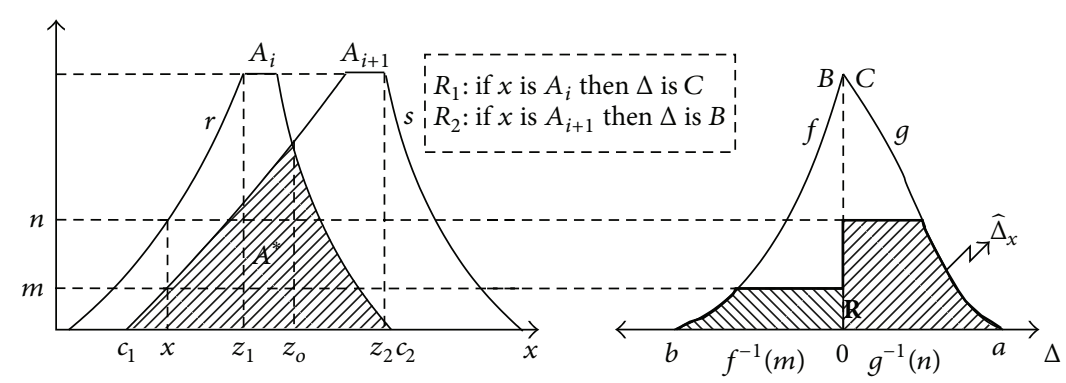

Figure 4: Mamdani's inference process for $A^{*}$ of the type $\left(A_{i}, A_{\mathrm{i}+1}\right) \rightarrow(C, B)$.

(ii) $\bigcap_{j=i, i+2} \operatorname{supp}\left(A_{j}\right)$ has at maximum only one element for each $1 \leq i<k-1$; that is, $\operatorname{supp}\left(A_{i}\right) \cap$ $\operatorname{supp}\left(A_{i+2}\right) \neq \phi$, if and only if, $\max \left\{x \in \operatorname{supp}\left(A_{i}\right)\right\}=$ $\min \left\{x \in \operatorname{supp}\left(A_{i+2}\right)\right\}$;

(iii) $\bigcup_{i=1, k} \operatorname{supp}\left(A_{i}\right)=U$, where $U$ is the domain of the fuzzy variable $x$;

(iv) given $z_{1} \in \operatorname{supp}\left(A_{i}\right)$ and $z_{2} \in \operatorname{supp}\left(A_{i+1}\right)$, if $A_{i}\left(z_{1}\right)=$ 1 and $A_{i+1}\left(z_{2}\right)=1$, then necessarily $z_{1}<z_{2}$ for each $1 \leq i<k$.

Definition 6. Consider a family of successive fuzzy subsets $\left\{A_{i}\right\}_{1 \leq i \leq k}$ that describe the antecedent of a fuzzy system associated with the p-fuzzy system (1). We say that $A^{*}$ is an equilibrium viable set of (1) if $A^{*}$ contains stationary points of (1).

If for some $1 \leq i<k$ there are $z_{1}, z_{2} \in\left[A_{i} \cup A_{i+1}\right]^{0}$ such that $\Delta\left(z_{1}\right) \cdot \Delta\left(z_{2}\right)<0$, then $A^{*}$ is given by $A^{*}=\left[A_{i} \cap A_{i+1}\right]^{0}$. If for all $z_{1}, z_{2} \in\left[A_{i} \cup A_{i+1}\right]^{0}, 1 \leq i<k, \Delta\left(z_{1}\right) \cdot \Delta\left(z_{2}\right)>0$, then $A^{*}=\left[A_{k}\right]^{0}$.

A p-fuzzy system depends on the fuzzy system associated with it, that is, it depends on the rule-base, on the inference method and on the defuzzification method used. In Definition 6, a sufficient condition for $\Delta\left(z_{1}\right) \cdot \Delta\left(z_{2}\right)<0$ is that the p-fuzzy system be associated with a fuzzy system whose rule-base in $A^{*}=\left[A_{i} \cup A_{i+1}\right]^{0}$ is of the type

$R_{1}$ : if $x$ is $A_{i}$ then $\Delta$ is $B$,

$R_{2}$ : if $x$ is $A_{i+1}$ then $\Delta$ is $C$,

where $\operatorname{supp}(B) \subset \mathbb{R}^{-}$and $\operatorname{supp}(C) \subset \mathbb{R}^{+}$or vice versa. When $\operatorname{supp}(B) \subset \mathbb{R}^{-}$and $\operatorname{supp}(C) \subset \mathbb{R}^{+}$we have that the equilibrium viable set $A^{*}$ is of the type $\left(A_{i}, A_{i+1}\right) \rightarrow(C, B)$. If, on the other hand, we have that $\operatorname{supp}(B) \subset \mathbb{R}^{+}$and $\operatorname{supp}(C) \subset \mathbb{R}^{-}$we say that $A^{*}$ is of the type $\left(A_{i}, A_{i+1}\right) \rightarrow$ $(B, C)$.

To understand the dynamics of the p-fuzzy system we need to understand how the rule-based system works more specifically, given $x \in A^{*}$, how is $\Delta(x)$ obtained? In the following section we will describe this process.

2.2. Output Defuzzification of the Fuzzy System. Let $A^{*}=$ $\left[A_{i} \cap A_{i+1}\right]^{0}=\left[c_{1}, c_{2}\right]$ be an equilibrium viable set of the p-fuzzy system. To facilitate the notation, we will indicate by $r$ the membership function of $A_{i}$, by $s$ the membership function of $A_{i+1}$,

$$
z_{1}=\min _{x \in \operatorname{supp}\left(A_{i}\right)}\{r(x)=1\}, \quad z_{2}=\max _{x \in \operatorname{supp}\left(A_{i+1}\right)}\{s(x)=1\},
$$

and by $f$ and $g$ the membership functions of $C$ and $D$ (Figure 4), respectively. Assume that the p-fuzzy system, in the equilibrium viable set $A^{*}$, is of the type $\left(A_{i}, A_{i+1}\right) \rightarrow$ $(C, B)$.

For each $x \in A^{*}, \Delta(x)$ is the $\mathbf{R}$ region centroid abscise, with $\mathbf{R}$ limited by the membership function of the fuzzy output, $\widehat{\Delta} x$, (see Figure 4). Thus,

$$
\begin{aligned}
\Delta(x)= & \left(\int_{b}^{f^{-1}(m)} t f(t) d t+\int_{f^{-1}(m)}^{0} m t d t+\int_{0}^{g^{-1}(n)} n t d t\right. \\
& \left.+\int_{g^{-1}(n)}^{a} t g(t) d t\right) \\
& \times\left(\int_{0}^{n} g^{-1}(t) d t-\int_{0}^{m} f^{-1}(t) d t\right)^{-1},
\end{aligned}
$$

where $(n, m)=(r(x), s(x))$. Equation (5) can be rewritten as

$$
\Delta(x)=\frac{h_{1}(n)+h_{2}(m)}{A(m, n)},
$$

where

$$
\begin{aligned}
& h_{1}(n)=\int_{0}^{g^{-1}(n)} n t d t+\int_{g^{-1}(n)}^{a} t g(t) d t, \\
& h_{2}(m)=\int_{b}^{f^{-1}(m)} t f(t) d t+\int_{f^{-1}(m)}^{0} m t d t, \\
& A(m, n)=\int_{0}^{n} g^{-1}(t) d t-\int_{0}^{m} f^{-1}(t) d t .
\end{aligned}
$$

2.3. Preliminary Results. In this section, we will present some important technical results for the main demonstrations of the theorems that establish sufficient conditions for uniqueness and existence of the stationary point of the p-fuzzy systems. Here, the presented results are technical enough and are important only for demonstrating the theorems in 
the next section. Therefore, the reader will be able, without loss of continuity, to skip them if desired.

The following results are referred to the equilibrium viable set of the type $\left(A_{i}, A_{i+1}\right) \rightarrow(C, B)$ (Figure 4$)$. From now on, it will be assumed that the functions $r, s, f$, and $g$ (which represent the membership functions $\mu_{A_{i}}, \mu_{A_{i+1}}, \mu_{B}$ and $\mu_{C}$, resp.) are continuous.

Lemma 7. The function $h_{1}(7)$ is increasing, and its range is given by, $\operatorname{Im}\left(h_{1}\right)=\left[0, \int_{0}^{a} \operatorname{tg}(t) d t\right]$.

Proof. Since $g$ is continuous in $[0, a]\left(g^{-1}\right.$ is limited in $\left.[0, a]\right)$, then the function $h_{1}$ is differentiable, and

$$
h_{1}^{\prime}(n)=\left(\int_{0}^{g^{-1}(n)} n t d t\right)^{\prime}+\left(\int_{g^{-1}(n)}^{a} \operatorname{tg}(t) d t\right)^{\prime}
$$

Using the derivative properties,

$$
\begin{gathered}
h_{1}^{\prime}(n)=\int_{0}^{g^{-1}(n)} t d t+n\left(\int_{0}^{g^{-1}(n)} t d t\right)^{\prime} \\
-\left(\int_{a}^{g^{-1}(n)} \operatorname{tg}(t) d t\right)
\end{gathered}
$$

and using the chain rule and the fundamental theorem of calculus, we obtain

$$
\begin{aligned}
h_{1}^{\prime}(n)= & \int_{0}^{g^{-1}(n)} t d t+n g^{-1}(n)\left(g^{-1}\right)^{\prime}(n) \\
& -n g^{-1}(n)\left(g^{-1}\right)^{\prime}(n) .
\end{aligned}
$$

Hence,

$$
h_{1}^{\prime}(n)=\int_{0}^{g^{-1}(n)} t d t=\frac{\left(g^{-1}(n)\right)^{2}}{2}>0 .
$$

Therefore, $h_{1}$ is increasing.

Since,

$$
\begin{gathered}
h_{1}(0)=\int_{0}^{g^{-1}(0)} 0 t d t+\int_{g^{-1}(0)}^{a} \operatorname{tg}(t) d t=\int_{a}^{a} \operatorname{tg}(t) d t=0, \\
h_{1}(1)=\int_{0}^{g^{-1}(1)} t d t+\int_{g^{-1}(1)}^{a} \operatorname{tg}(t) d t=\int_{0}^{a} \operatorname{tg}(t) d t,
\end{gathered}
$$

then $\operatorname{Im}\left(h_{1}\right)=\left[0, \int_{0}^{a} \operatorname{tg}(t) d t\right]$.

Lemma 8. The function $h_{2}$ is decreasing, and its range is given by $\operatorname{Im}\left(h_{2}\right)=\left[\int_{b}^{0} t f(t) d t, 0\right]$.

Proof. Analogous to the demonstration of Lemma 7.

Lemma 9. Let $\phi: I=\left[d_{1}, d_{2}\right] \rightarrow \mathbb{R}$ be a function of the class $C^{2}$. If $\phi^{\prime \prime}(z)>0, \forall z \in\left(d_{1}, d_{2}\right)$ and $\phi\left(d_{1}\right)<0$, then $\phi$ has at maximum one root in $I$.

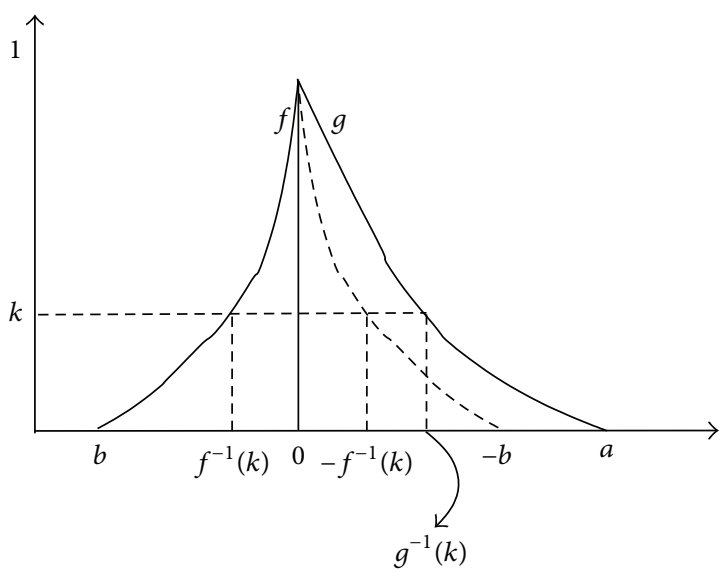

FIGURE 5: System p-fuzzy output with $g(t)>f(-t)$.

Proof of Lemma 9. Assume there are $z_{1}, z_{2} \in I\left(z_{1}<z_{2}\right)$ such that $\phi\left(z_{1}\right)=\phi\left(z_{2}\right)=0$. Since $\phi^{\prime \prime}(z)>0$, we have that $\phi$ is not constant. Hence, by Rolle's Theorem, $\exists c \in\left(z_{1}, z_{2}\right)$ such that $\phi^{\prime}(c)=0$. Hence $c$ is a minimum point, because $\phi^{\prime \prime}(c)>0$. But, $\phi\left(d_{1}\right)<0 \Rightarrow \phi(c)>0$. Since $\phi$ is continuous $\exists z_{o} \in$ $\left(z_{1}, z_{2}\right)$ such that $\phi(c)>\phi\left(z_{o}\right)>0$, it is nonsense! Therefore $\phi$ has at maximum one root.

Lemma 10. If $g(t)>f(-t), \forall t \in[0,-b]$, then $g^{-1}(k)>$ $-f^{-1}(k) \forall k \in[0,1]$.

Proof. The proof is simple (see Figure 5).

Lemma 11. If $g(t)>f(-t), \forall t \in[0,-b]$ then for $m, n \in[0,1]$ with $m \leq n$ one has that

$$
\Delta(x)=\frac{h_{1}(n)+h_{2}(m)}{A(m, n)}>0 .
$$

Proof. The proof is simple.

Lemma 12. If $g(t)<f(-t), \forall t \in[0, a]$, and $m, n \in[0,1] m \geq$ $n$, then $\Delta(x)<0$.

Proof. It is analogous to the proof of Lemma 11.

\section{Stationary Point}

In this section, we will enunciate and prove a theorem that guarantees the existence of at least one stationary point for each equilibrium viable set of the $\mathrm{p}$-fuzzy system. For this, we will use again Figure 4 to motivate the presented results in this section.

Theorem 13 (existence). Let $S$ be a $p$-fuzzy system and $A^{*}$ an equilibrium viable set of $S$ of the type $\left(A_{i}, A_{i+1}\right) \rightarrow(C, B)$. Then, there is at least one stationary point of $S$ in $A^{*}$. That is, $\exists x^{*} \in A^{*}$ such that $\Delta\left(x^{*}\right)=0$. 
Proof. Given $x \in A^{*}$, from Definition 4, then $x$ is a stationary point if and only if

$$
\Delta(x)=0 \Longleftrightarrow h_{1}(n)+h_{2}(m)=0 .
$$

If $\mu_{A_{i}}\left(c_{1}\right)=0$, then

$$
\Delta\left(c_{1}\right)=h_{1}\left(\mu_{A_{i}}\left(c_{1}\right)\right)+h_{2}\left(\mu_{A_{i+1}}\left(c_{1}\right)\right)=h_{1}(0)+h_{2}(0)=0,
$$

and, therefore $c_{1}$ is a stationary point. If $\mu_{A_{i+1}}\left(c_{2}\right)=0$ one has that $\Delta\left(c_{2}\right)=0$; hence $c_{2}$ is a stationary point. Now, assume that $\mu_{A_{i}}\left(c_{1}\right)>0$ and $\mu_{A_{i+1}}\left(c_{2}\right)>0$. Since $\mu_{A_{i+1}}\left(c_{1}\right)=0$, then from Lemmas 7 and 8 one has that $h_{1}\left(\mu_{A_{i}}\left(c_{1}\right)\right)>0$ and $h_{2}\left(\mu_{A_{i+1}}\left(c_{1}\right)\right)=0$. Therefore

$$
\Delta\left(c_{1}\right)=h_{1}\left(\mu_{A_{i}}\left(c_{1}\right)\right)+h_{2}\left(\mu_{A_{i+1}}\left(c_{1}\right)\right)=h_{1}\left(\mu_{A_{i}}\left(c_{1}\right)\right)>0 .
$$

Thus, from Lemma 7,

$$
\Delta\left(c_{2}\right)=h_{1}\left(\mu_{A_{i}}\left(c_{2}\right)\right)+h_{2}\left(\mu_{A_{i+1}}\left(c_{2}\right)\right)=h_{2}\left(\mu_{A_{i+1}}\left(c_{2}\right)\right)<0 .
$$

Since $\Delta$ is continuous, by Bolzano's Intermediate Value Theorem, $\exists x^{*} \in\left[c_{1}, c_{2}\right]$ such that $\Delta\left(x^{*}\right)=0$; therefore, $x^{*}$ is a stationary point.

Remark 14. If in Theorem 13 we consider $A^{*}$ as an equilibrium viable set of the type $\left(A_{i}, A_{i+1}\right) \rightarrow(B, C)$, the result is analogous; that is, there exists a stationary point $x^{*} \in A^{*}$.

3.1. Local Stationary Points-Symmetrical Output. If $A^{*}$ is an equilibrium viable set, where the membership functions of the consequents, $B$ and $C$, are symmetrical functions, then the stationary point in $A^{*}$ is unique. Except when $\mu_{A_{i}}\left(c_{1}\right)=0$ or $\mu_{A_{i+1}}\left(c_{2}\right)=0$ possibly occurs. Then we have the following proposition.

Proposition 15. Let $S$ be a p-fuzzy system and $A^{*}$ an equilibrium viable set of $S$ of the type $\left(A_{i}, A_{i+1}\right) \rightarrow(C, B)$. If the membership functions of $B$ and $C$, respectively, $\mu_{B}$ and $\mu_{C}$, are monotonous and symmetric, that is, $\mu_{C}(t)=\mu_{B}(-t)$, then there exists an equilibrium point in $A^{*}$ :

$$
x^{*}=\max _{x \in A^{*}}\left[\min \left(\mu_{A_{i}}(x), \mu_{A_{i+1}}(x)\right)\right] .
$$

Proof. Since $\mu_{B}(t)=\mu_{C}(-t)$ then $\mu_{B}(-a)=\mu_{C}(a)=0=$ $\mu_{B}(b) \Rightarrow b=-a$, because $\mu_{B}$ U' is monotonous. Yet, we have that $\mu_{B}(t)=\mu_{C}(-t) \Rightarrow \mu_{B}^{-1}\left(\mu_{C}(t)\right)=-t=-\mu_{B}^{-1}\left(\mu_{B}(t)\right) \Rightarrow$ $\mu_{B}^{-1}(y)=-\mu_{C}^{-1}(y)$.

Then, $\Delta\left(z_{0}\right)=0$, if and only if, $h_{1}(n)=-h_{2}(m)$. Since $b=-a$, from (8), then we obtain

$$
h_{2}(m)=\int_{-a}^{\mu_{B}^{-1}(m)} t \mu_{B}(t) d t+\int_{\mu_{B}^{-1}(m)}^{0} m t d t
$$

If we perform a change in the variable $u=-t$, we have

$$
\begin{gathered}
h_{2}(m)=\int_{a}^{-\mu_{B}^{-1}(m)} u \mu_{B}(-u) d u+\int_{-\mu_{B}^{-1}(m)}^{0} m u d u \\
\Longrightarrow h_{2}(m)=\int_{a}^{\mu_{C}^{-1}(m)} u \mu_{C}(u) d u+\int_{\mu_{C}^{-1}(m)}^{0} m u d u=-h_{1}(m) .
\end{gathered}
$$

That is, $h_{2}=-h_{1}$. Hence, $h_{1}(n)=-h_{2}(m) \Leftrightarrow h_{1}(n)=$ $h_{1}(m) \Leftrightarrow m=n$ (because $h_{1}$ is increasing: Lemma 7); that proves the proposition.

Remark 16. If $\mu_{A_{i}}\left(c_{1}\right) \neq 0$, then $x^{*}=\max _{x \in A^{*}}\left[\min \left(\mu_{A_{i}}(x)\right.\right.$, $\left.\left.\mu_{A_{i+1}}(x)\right)\right]$ is the only stationary point in $A^{*}$. Besides that, if the system $S$ is of the type $\left(A_{i}, A_{i+1}\right) \rightarrow(B, C)$, then the result of Proposition 15 is the same.

\section{Uniqueness of the Stationary Point}

In this section we will enunciate and prove theorems that establish condition for uniqueness of the stationary point of a one-dimensional p-fuzzy system. Initially we will consider a simpler case, when $A^{*} \subset\left[z_{1}, z_{2}\right]$ (Figure 4 ), where

$$
z_{1}=\min _{x \in \operatorname{supp}\left(A_{i}\right)}\{r(x)=1\}, \quad z_{2}=\max _{x \in \operatorname{supp}\left(A_{i+1}\right)}\{s(x)=1\} .
$$

Theorem 17. Let $S$ be a p-fuzzy system and $A^{*}$ an equilibrium viable set of $S$ of the type $\left(A_{i}, A_{i+1}\right) \rightarrow(C, B)$. If the functions $\mu_{A_{i}}$ and $\mu_{A_{i+1}}$ are piecewise monotonous and $A^{*} \subset\left[z_{1}, z_{2}\right]$ then there exists only one stationary point in $A^{*}$.

Proof. Given $x \in A^{*}$ one has

$$
\Delta(x)=h_{1}(n)+h_{2}(m)=h_{1}\left(\mu_{A_{i}}(x)\right)+h_{2}\left(\mu_{A_{i+1}}(x)\right) .
$$

Using Lemmas 7 and 8 and the chain rule we find that the derivative of $\Delta$ is

$$
\begin{aligned}
\Delta^{\prime}(x)= & \frac{\left[\mu_{C}^{-1}\left(\mu_{A_{i}}(x)\right)\right]^{2}}{2} \mu_{A_{i}}^{\prime}(x) \\
& -\frac{\left[\mu_{B}^{-1}\left(\mu_{A_{i+1}}(x)\right)\right]^{2}}{2} \mu_{A_{i+1}}^{\prime}(x) .
\end{aligned}
$$

Since in $A^{*}=\left[c_{1}, c_{2}\right] \mu_{A_{i}}$ is not increasing and $\mu_{A_{i+1}}$ is not decreasing, then $\mu_{A_{i}}^{\prime}(x) \leq 0$ and $\mu_{A_{i+1}}^{\prime}(x) \geq 0$, and, besides that, if $\mu_{A_{i}}^{\prime}(x)=0$, we have that $\mu_{A_{i+1}}^{\prime}(x) \neq 0$ and if $\mu_{A_{i+1}}^{\prime}(x)=$ 0 we obtain $\mu_{A_{i}}^{\prime}(x) \neq 0$. Then, from (25), $\Delta^{\prime}(x)<0$. This shows that $\Delta$ is decreasing. From Theorem 13, there exists a stationary point in $A^{*}$ then this point is unique.

Now, consider the more general case, when $A^{*} \not \subset\left[z_{1}, z_{2}\right]$, and divide it into two theorems. Initially, consider the case that the membership functions $\mu_{C}$ and $\mu_{B}$ are such that $\mu_{C}(t)>\mu_{B}(-t)$; next, consider the case where $\mu_{C}(t)<$ $\mu_{B}(-t)$. 


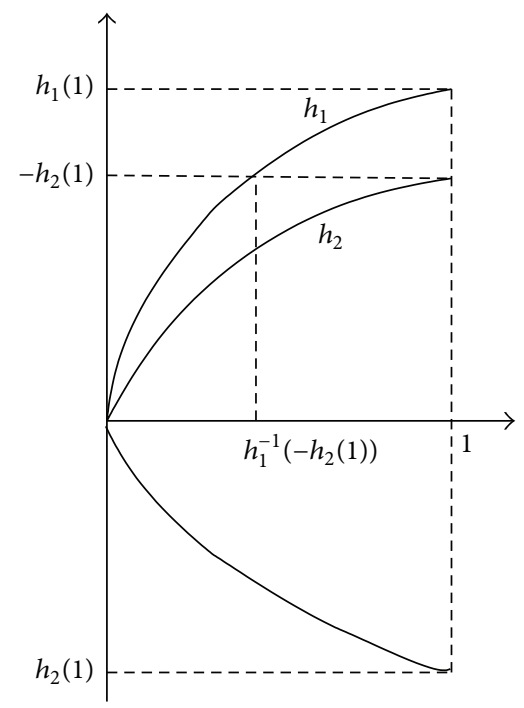

FIgURE 6: Functions $h_{1}$ and $h_{2}$.

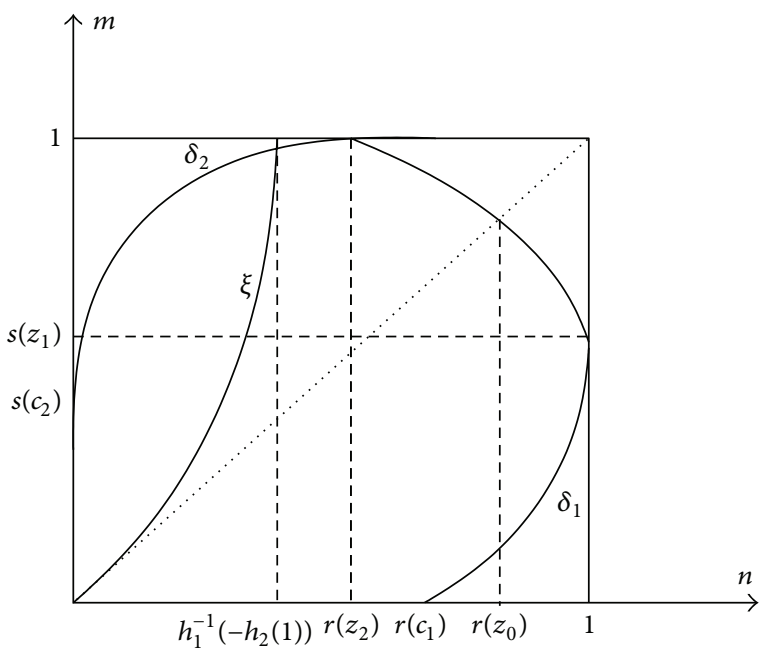

Figure 7: Functions $\xi, \delta_{1}$ and $\delta_{2}$.

4.1. Case 1: $\mu_{C}(t)>\mu_{B}(-t)$

Theorem 18 (uniqueness). Let $S$ be a $p$-fuzzy system and $A^{*}$ an equilibrium viable set of $S$ of the type $\left(A_{i}, A_{i+1}\right) \rightarrow$ $(C, B)$. If the functions $\mu_{A_{i}}, \mu_{A_{i+1}}, \mu_{B}$ and $\mu_{C}$ are continuously differentiable, $\mu_{A_{i}}$ and $\mu_{A_{i+1}}$ are piecewise monotonous $\mu_{B}$ and $\mu_{\mathrm{C}}$ are strictly monotonous, such that

(i) $\mu_{C}(t)>\mu_{B}(-t), \forall t \in(0,-b)$,

(ii) $\mu_{C}^{\prime}(q) / \mu_{B}^{\prime}(p)<\left(p^{3} / q^{3}\right), \forall p \in \operatorname{supp}(B), q \epsilon$ $\operatorname{supp}(C)$ and $\mu_{B}(p)>\mu_{C}(q)$,

(iii) $\left[\mu_{A_{i+1}}^{\prime}(x) / \mu_{A_{i}}^{\prime}(x)\right] \leq 0, \forall x \in\left(z_{o}, c_{2}\right), \mu_{A_{i}}(x) \neq \mu_{A_{i+1}}(x)$.

Then, $S$ has only one stationary point, $x^{*}$ in $A^{*}$, and $x^{*} \epsilon$ $\left(z_{o}, c_{2}\right]$.

Proof. For the sake of simplicity notation, we make $r=\mu_{A_{i}}$, $s=\mu_{A_{i+1}}, f=\mu_{B}$ and $g=\mu_{C}$.
Initially, we see that given $x \in\left(z_{0}, c_{2}\right]$ (Figure 4), $x$ determines only one $(n, m) \in[0,1]^{2}$ such that $n=r(x)$ and $m=s(x)$. By monotonicity of $r$, we have that for each $n \in\left[0, r\left(z_{o}\right)\right)$ there exists only one $m \in[0,1]$ such that $n=r(x)$ and $m=s(x)$. That is, each $(n, m)$, in this situation, determines only one $x \in\left(z_{o}, c_{2}\right]$.

By Theorem 13, there exists a stationary point $x^{*} \epsilon$ $\left[c_{1}, c_{2}\right]=\left[c_{1}, z_{o}\right] \cup\left(z_{o}, c_{2}\right]$. Given $x \in\left[c_{1}, z_{o}\right] \Rightarrow m=s(x) \leq$ $n=r(x)$. Then, by Lemma $11 x^{*} \notin\left[c_{1}, z_{0}\right] \Rightarrow x^{*} \in\left(z_{0}, c_{2}\right]$. That is equivalent to the existence of only one $\left(n^{*}, m^{*}\right)$, with $n^{*} \in\left[0, r\left(z_{o}\right)\right)$ such that $H\left(n^{*}, m^{*}\right)=0$.

Since for each $n \in\left[0, r\left(z_{o}\right)\right)$ there exists only one $m \in$ $[0,1]$ such that $n=r(x)$ and $m=s(x)$, then we may define a function $\delta_{2}:\left[0, r\left(z_{o}\right)\right) \rightarrow[0,1]$ such that $m=\delta_{2}(n)$ (Figure 7). We observe that $\delta_{2}$ is continuous, because $r$ and $s$ are continuous. Using the chain rule we get the derivative of $\delta_{2}$ to be

$$
\delta_{2}^{\prime}(n)=\frac{s^{\prime}(x)}{r^{\prime}(x)} \stackrel{(\mathrm{iii})}{\Rightarrow} \delta_{2}^{\prime \prime}(n) \leq 0, \quad \forall n \in D_{\delta_{2}}
$$

By Lemmas 7 and $8, h_{1}$ and $-h_{2}$ are increasing, and, by condition (i), it follows that (Figure 6)

$$
\int_{0}^{a} \operatorname{tg}(t) d t>-\int_{b}^{0} t f(t) d t \Longleftrightarrow h_{1}(1)>-h_{2}(1) .
$$

Then, given $n \in\left[0, h_{1}^{-1}\left(-h_{2}(1)\right)\right]$, there exists only one $m \in$ $[0,1]$ such that

$$
h_{1}(n)=-h_{2}(m) \Longleftrightarrow h_{1}(n)+h_{2}(m)=0 .
$$

Therefore, we may define an injective function $\xi, m=\xi(n)$ (Figure 7) so that the inverse range of 0 by $H$ is given by

$$
H^{-1}(0)=\{(n, m) ; m=\xi(n)\},
$$

where $H:\left[0, h_{1}^{-1}\left(-h_{2}(1)\right)\right] \times[0,1] \rightarrow \mathbb{R}$ is given by $H(n, m)=h_{1}(n)+h_{2}(m)$.

Since $\partial H / \partial n=h_{1}^{\prime}(n)=\left(g^{-1}(n)\right)^{2} / 2>0($ Lemma 7$)$ and $\partial H / \partial m=h_{2}^{\prime}(m)=-\left(f^{-1}(m)\right)^{2} / 2<0$ (Lemma 8) then, by the Implicity Function Theorem, $\xi$ is $k$ times differentiable, and, besides that,

$$
\begin{gathered}
\xi^{\prime}(n)=-\frac{d h_{1} / n}{d h_{2} / m}=\left[\frac{g^{-1}(n)}{f^{-1}(m)}\right]^{2}>0, \\
\forall n \in\left(0, h_{1}^{-1}\left(-h_{2}(1)\right)\right), m \in(0,1), m=\xi(n) .
\end{gathered}
$$

Thus, $\xi$ is a strictly increasing function, and since $H(0,0)=0$ and $H\left(h_{1}^{-1}\left(-h_{2}(1)\right), 1\right)=0$, then $D_{\xi}=\left[0, h_{1}^{-1}\left(h_{2}(1)\right)\right]$ and $\operatorname{Im}_{\xi}=[0,1]$.

Given $m, n \in(0,1)$, there is only one $p \in(b, 0)$ such that $p=f^{-1}(m)$, and there exists only one $q \in(0, a)$ such that $q=$ $g^{-1}(n)$, since by assumption $f$ and $g$ are strictly monotonous.

From Lemma 11 we have that $m \leq n \Rightarrow H(m, n)>0$. Hence, $H(m, n)=0 \Rightarrow m>n$. Therefore, we are interested in the pairs $(m, n)$ such that $m>n$. Thus, we have

$$
m>n \Longleftrightarrow f(p)>g(q) .
$$




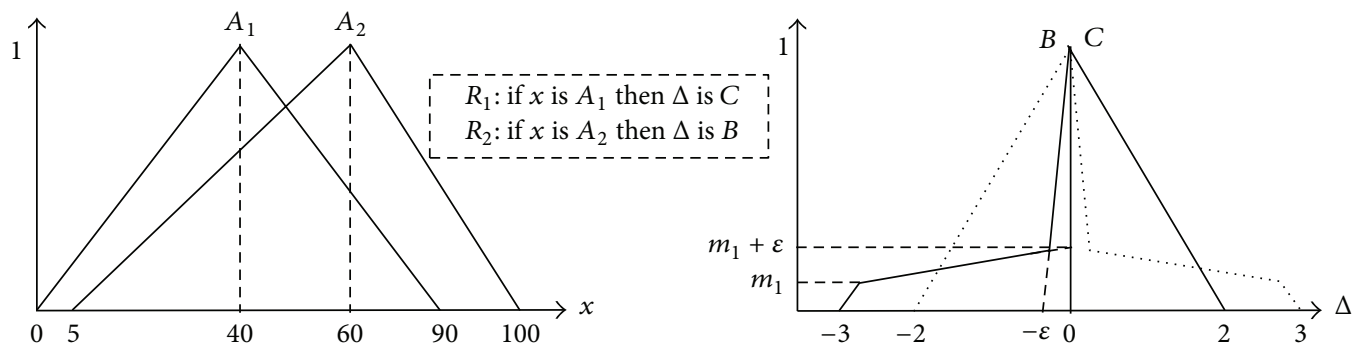

FIGURE 8: p-Fuzzy system: existence of more than one stationary point.

Since $f$ and $g$ are monotonous, by Lagrange's Medium Value Theorem, we obtain

$$
\begin{gathered}
p=f^{-1}(m) \Longleftrightarrow\left(f^{-1}\right)^{\prime}(m)=\frac{1}{f^{\prime}(p)} \\
q=g^{-1}(n) \Longleftrightarrow\left(g^{-1}\right)^{\prime}(n)=\frac{1}{g^{\prime}(q)} .
\end{gathered}
$$

Then,

$$
\begin{aligned}
& m>n \stackrel{(31)}{\Longleftrightarrow} f(p)>g(q) \stackrel{(\mathrm{ii})}{\Rightarrow} \frac{g^{\prime}(q)}{f^{\prime}(p)} \\
&<\frac{p^{3}}{q^{3}} \stackrel{(32) e(33)}{\Longleftrightarrow} \frac{\left(f^{-1}\right)^{\prime}(m)}{\left(g^{-1}\right)^{\prime}(n)}<\frac{\left[f^{-1}(m)\right]^{3}}{\left[g^{-1}(n)\right]^{3}} .
\end{aligned}
$$

Therefore,

$$
\begin{aligned}
m>n & \Longrightarrow\left(f^{-1}\right)^{\prime}(m)\left[g^{-1}(n)\right]^{3} \\
& -\left(g^{-1}\right)^{\prime}(n)\left[f^{-1}(m)\right]^{3}>0 .
\end{aligned}
$$

By differentiation of (30), we obtain

$$
\begin{aligned}
\xi^{\prime \prime}(n)= & \frac{-2 g^{-1}(n)}{\left[f^{-1}(m)\right]^{5}} \\
& \times\left\{\left(f^{-1}\right)^{\prime}(m)\left[g^{-1}(n)\right]^{3}-\left(g^{-1}\right)^{\prime}(n)\left[f^{-1}(m)\right]^{3}\right\}
\end{aligned}
$$

and since $-2 g^{-1}(n) /\left[f^{-1}(m)\right]^{5}>0, \forall m, n \in(0,1)$, from (35), we have

$$
\xi^{\prime \prime}(n)>0, \quad \forall n \in \stackrel{o}{D_{\xi}} .
$$

Now we take $I=D_{\xi} \cap D_{\delta_{2}}=D_{\xi} \cap\left[0, r\left(z_{o}\right)\right)$, and we define the function $\phi: I \rightarrow[0,1]$ such that

$$
\phi(n)=\xi(n)-\delta_{2}(n) .
$$

Then, from (26) and (37) we have $\phi^{\prime \prime}(n)>0, \forall n \stackrel{o}{\in}$. Since $\xi(0)=0$ and by condition (iii) of Theorem 18, it follows that $\delta_{2}(0)>0$; then we have $\phi(0)<0$. Consequently, from Lemma 9 , we have that there is only one $n^{*} \in I$ such that

$$
\phi\left(n^{*}\right)=0 \stackrel{(38)}{\Longleftrightarrow} \xi\left(n^{*}\right)=\delta_{2}\left(n^{*}\right) .
$$

Since $\xi=H^{-1}(0)$, then we obtain

$$
0=H\left(n^{*}, \xi\left(n^{*}\right)\right) \stackrel{(39)}{=} H\left(n^{*}, \delta_{2}\left(n^{*}\right)\right) .
$$

So, there exists only one $x^{*} \in\left(z_{o}, c_{2}\right], n^{*}=r\left(x^{*}\right)$ and $m^{*}=\delta_{2}\left(n^{*}\right)=s\left(x^{*}\right)$ such that

$$
\Delta\left(x^{*}\right)=\frac{H\left(n^{*}, m^{*}\right)}{A\left(n^{*}, m^{*}\right)}=0 .
$$

This finally proves the theorem.

\subsection{Case 2: $\mu_{C}(t)<\mu_{B}(-t)$}

Theorem 19 (uniqueness). Let $S$ be a p-fuzzy system and $A^{*}$ an equilibrium viable set of $S$ of the type $\left(A_{i}, A_{i+1}\right) \rightarrow$ $(C, B)$. If the functions $\mu_{A_{i}}, \mu_{A_{i+1}}, \mu_{B}$, and $\mu_{C}$ are continuously differentiable, $\mu_{A_{i}}$ and $\mu_{A_{i+1}}$ are piecewise monotonous, $\mu_{B}$ and $\mu_{C}$ are strictly monotonous, such that

(i) $\mu_{C}(t)<\mu_{B}(-t), \forall t \in(0, a)$,

(ii) $\mu_{C}^{\prime}(q) / \mu_{B}^{\prime}(p)>p^{3} / q^{3}, \forall p \in \operatorname{supp}(B), q \in \operatorname{supp}(C)$ and $\mu_{B}(p)<\mu_{C}(q)$,

(iii) $\left[\mu_{A_{i}}^{\prime}(x) / \mu_{A_{i+1}}^{\prime}(x)\right] \leq 0, \forall x \in\left(c_{1}, z_{o}\right), \mu_{A_{i}}(x) \neq \mu_{A_{i+1}}(x)$.

Then, $S$ has only one stationary point, $x^{*}$ in $A^{*}$, and $x^{*} \in$ $\left[c_{1}, z_{o}\right)$.

Proof. It is analogous to the proof of Theorem 18.

4.3. Some Comments about Uniqueness Theorems. When we do not have $\mu_{C}(t)>\mu_{B}(-t)$ or $\mu_{C}(t)<\mu_{B}(-t)$, it is impossible to establish general conditions for uniqueness of stationary points. For example, consider the p-fuzzy system in Figure 8. This system has an equilibrium viable set, $A^{*}=\left[A_{1} \cap A_{2}\right]^{0}$. 


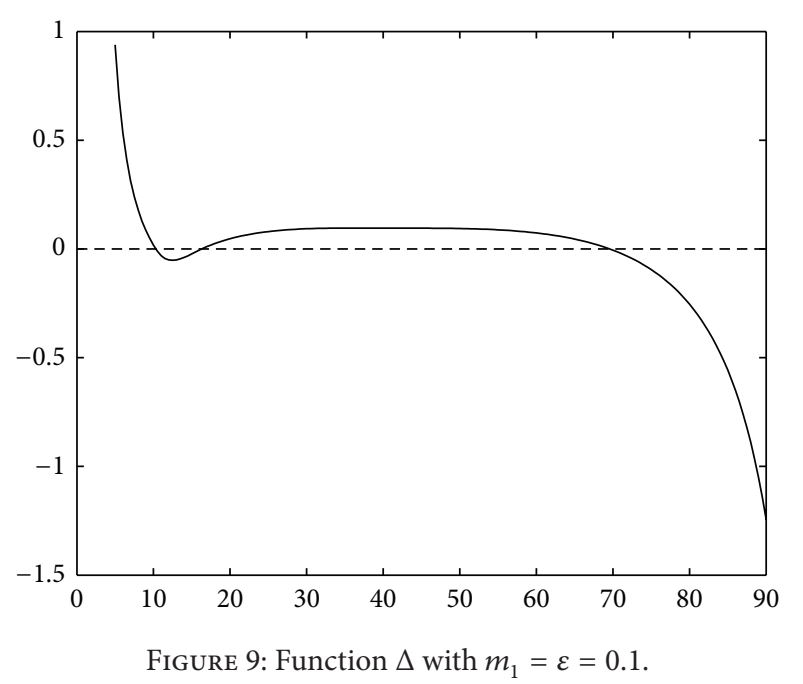

The sets that describe the input variable have membership functions $\mu_{A_{1}}$ and $\mu_{A_{2}}$ :

$$
\begin{aligned}
& \mu_{A_{1}}(x)= \begin{cases}\frac{1}{40} x, & \text { if } 0<x \leq 40 \\
\frac{-1}{50} x+\frac{9}{5}, & \text { if } 40<x \leq 90 \\
0, & \text { otherwise, }\end{cases} \\
& \mu_{A_{2}}(x)= \begin{cases}\frac{1}{55} x-\frac{1}{11}, & \text { if } 5<x \leq 60 \\
\frac{-1}{40} x+\frac{5}{2}, & \text { if } 60<x \leq 100 \\
0, & \text { otherwise, }\end{cases}
\end{aligned}
$$

and the fuzzy sets that describe the output variable have membership functions: $\mu_{C}(t)=(-1 / 2) t+1$ and

$$
\begin{aligned}
& \mu_{B}(t) \\
& =\left\{\begin{array}{lc}
\frac{1}{3} t+1, & \text { if }-3<t \leq 3\left(m_{1}-1\right) \\
\frac{\varepsilon}{3\left(m_{1}-1\right)} t+m_{1}+\varepsilon, & \text { if } 3\left(m_{1}-1\right)<t \\
& \leq \frac{3 \varepsilon\left(\varepsilon+m_{1}-1\right)\left(m_{1}-1\right)}{3\left(m_{1}-1\right)+\varepsilon^{2}} \\
\frac{1}{\varepsilon} t+1, & \text { if } \frac{3 \varepsilon\left(\varepsilon+m_{1}-1\right)\left(m_{1}-1\right)}{3\left(m_{1}-1\right)+\varepsilon^{2}} \\
0, & <t \leq 0
\end{array}\right. \\
& \text { otherwise. }
\end{aligned}
$$

For example, if we take in (43) $m_{1}=0.1$ and $\varepsilon=0.1$, the p-fuzzy system obtained has three stationary points in $A^{*}$, which can be visualized in Figure 9, which depicts the graphic of function $\Delta$.

Remark 20. Note that the function $\mu_{B}$ is not differentiable in all points into $\operatorname{supp}(B)$, which was a requirement made in the previous cases. However, it can be clearly constructed a function $\mu_{B}$ derivable in all points of $\operatorname{supp}(B)$. For example,

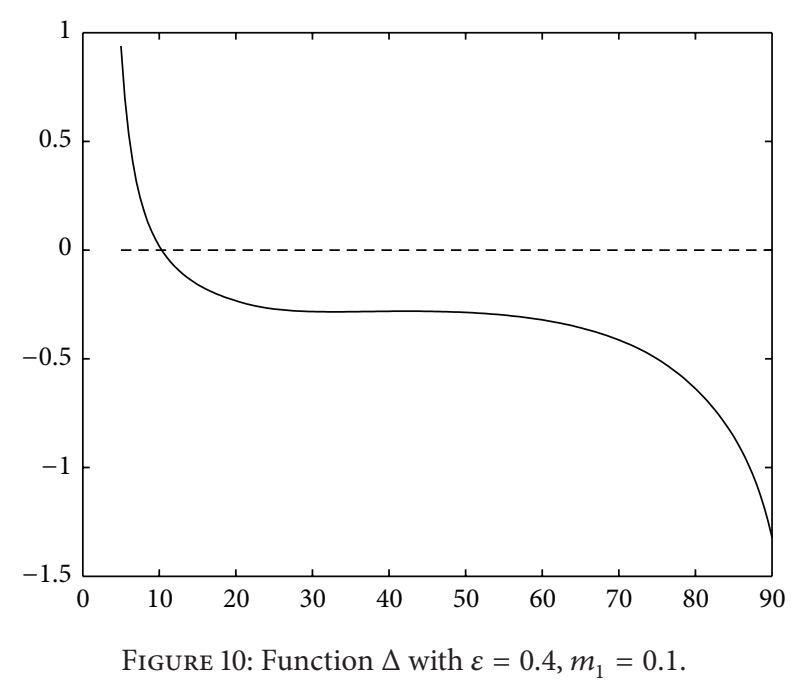

if we substitute the second sentence of $\mu_{B}$ by an adequate fourth degree polynomial, obviously $\mu_{B}$ will be derivable into $\operatorname{supp}(B)$.

Remark 21. If we take, for example, $\varepsilon=0.3$ and $m_{1}=$ 0.3 , we have that the obtained p-fuzzy system has only one stationary point (Figure 10). This shows that Theorems 18 and 19 establish only sufficient conditions for uniqueness of the stationary point.

4.4. Uniqueness for Triangular and Trapezoidal Membership Functions. In this section, we will list some important consequences of Theorems 18 and 19. We will also show that for triangular and trapezoidal membership functions, the stationary point is only one in $A^{*}$. However, before doing so, let us take a look at the following lemmas.

Lemma 22. If $\mu_{B}(p)>\mu_{C}(q)$ then $q>-p$, where $p=\mu_{B}^{-1}(m)$ and $q=\mu_{C}^{-1}(n)$.

Proof. In fact, we have that $\mu_{B}(p)>\mu_{C}(q) \Rightarrow m>n$ hence using Lemma 10 and the fact that $-\mu_{B}^{-1}$ isincreasing, once $\mu_{B}$ is increasing, then we have that

$$
q=\mu_{C}^{-1}(n)>-\mu_{B}^{-1}(n)>-\mu_{B}^{-1}(m)=-p .
$$

Lemma 23. If $\mu_{B}(p)<\mu_{C}(q)$, then $q<-p$, where $p=\mu_{B}^{-1}(m)$ and $q=\mu_{C}^{-1}(n)$.

Proof. Analogous to previous proof.

Corollary 24. Let $S$ be a p-fuzzy system and $A^{*}$ an equilibrium viable set of S. If $\mu_{A_{i}}, \mu_{A_{i+1}}, \mu_{B}$ and $\mu_{C}$ are triangular fuzzy numbers, then $S$ has only one stationary point in $A^{*}$.

Proof. We will prove the case where $S$ is $\left(\mu_{A_{i}}, \mu_{A_{i+1}}\right) \rightarrow$ $\left(\mu_{C}, \mu_{B}\right)$. If $S$ is $\left(\mu_{A_{i}}, \mu_{A_{i+1}}\right) \rightarrow\left(\mu_{B}, \mu_{C}\right)$, then the proof is analogous. 


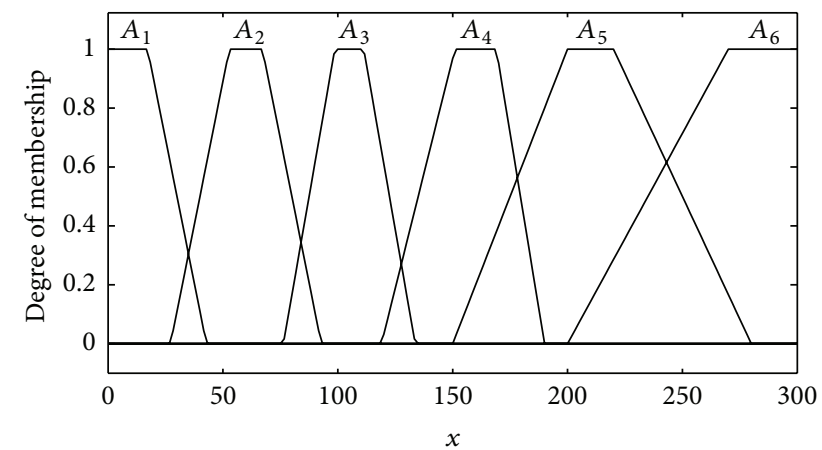

FIgURE 11: Population $(x)$.

If $a=b$, then $\mu_{A_{i}}$ and $\mu_{A_{i+1}}$ are symmetrical and by Proposition 15 the stationary point is only one:

$$
x^{*}=\max _{x \in A^{*}}\left[\min \left(\mu_{A_{i}}(x), \mu_{A_{i+1}}(x)\right)\right] .
$$

Assume that $a>b$; then $\mu_{A_{i}}, \mu_{A_{i+1}}, \mu_{B}$, and $\mu_{C}$ satisfy Theorem 18. In fact, (i) and (iii) are trivial. Since $\mu_{B}(t)=$ $-(1 / b) t+1$ and $\mu_{C}(t)=-(1 / a) t+1$, then $\mu_{B}(p)>\mu_{C}(q) \Rightarrow$ $b / a<p / q \Rightarrow \mu_{C}^{\prime}(q) / \mu_{B}^{\prime}(p)<p / q$. From Lemma 22, we have that $q>-p \Rightarrow p / q<p^{3} / q^{3}$ and, therefore, we obtain $\mu_{C}^{\prime}(q) / \mu_{B}^{\prime}(p)<p^{3} / q^{3}$, which satisfies (ii).

Now, assume that $a<b$; then $\mu_{A_{i}}, \mu_{A_{i+1}}, \mu_{B}$, and $\mu_{C}$ satisfy the Theorem 19. In fact, (i) and (iii) are trivial and $\mu_{B}(p)<\mu_{C}(q) \Rightarrow b / a>p / q \Rightarrow \mu_{C}^{\prime}(q) / \mu_{B}^{\prime}(p)>p / q$. From Lemma 23 we get $q<-p \Rightarrow p / q>p^{3} / q^{3}$, and, therefore, $\mu_{C}^{\prime}(q) / \mu_{B}^{\prime}(p)>p^{3} / q^{3}$, which satisfies (ii). This concludes the proof.

Corollary 25. Let $S$ be a $p$-fuzzy system and $A^{*}$ an equilibrium viable set of $S$. If $\mu_{A_{i}}$ and $\mu_{A_{i+1}}$ are trapezoidal fuzzy numbers and $\mu_{B}$ and $\mu_{C}$ are triangular fuzzy numbers, then $S$ has only one stationary point in $A^{*}$.

Proof. It is analogous to the proof of Corollary 24.

\section{Examples}

In this section we will present some computational experiments that confirm the mathematical theory presented in the previous sections. The experiments had been carried out with Matlab software. For the experiments we will consider inhibited one-dimensional p-fuzzy systems. These systems can be used to model situations where the state variable is increasing (resp., decreasing) with a carrying capacity (resp. lower bound). These situations, in population dynamics, are described by inhibited models such as Gompertz's model, Verhulst's model, von Bertallanffy's models, and Asymptotic Exponential model.

The inhibited one-dimensional p-fuzzy systems are composed of the variables "Population" (Figure 11) and "Variation" (Figure 12). The rule-base of these systems is

(1) if Population is low $\left(A_{1}\right)$ then Variation is low positive $(C)$;

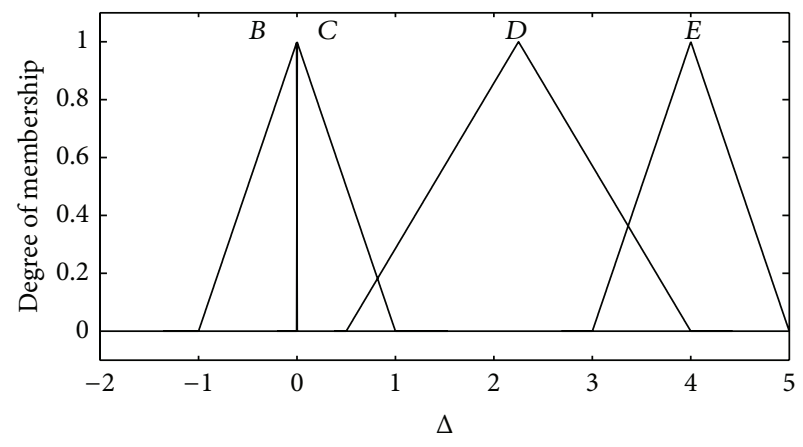

Figure 12: Variation $(\Delta): \mu_{C}(t)=\mu_{B}(-t)$.

(2) if Population is medium low $\left(A_{2}\right)$ then Variation is medium positive $(D)$;

(3) if Population is medium $\left(A_{3}\right)$ then Variation is high positive $(E)$;

(4) if Population is medium high $\left(A_{4}\right)$ then Variation is medium positive $(D)$;

(5) if Population is high $\left(A_{5}\right)$ then Variation is low positive $(C)$;

(6) if Population is the highest $\left(A_{6}\right)$ then Variation is low negative $(B)$.

5.1. Example 1. In this system the membership functions of $B$ and $C$ are $\mu_{B}(t)=t+1$ and $\mu_{C}(t)=1-t$. These functions are symmetric (Figure 12). Observing the rules we can identify an equilibrium viable set, $A^{*}=[200,280]$, where $A^{*}=A_{5} \cap A_{6}$, in which membership functions are given by size:

$$
\mu_{A_{5}}(x)= \begin{cases}\frac{1}{50}(x-150), & \text { if } 150<x \leq 200 \\ 1, & \text { if } 200<x \leq 220 \\ \frac{-1}{60}(x-280), & \text { if } 220<x \leq 270 \\ 0, & \text { otherwise, }\end{cases}
$$

$$
\mu_{A_{6}}(x)= \begin{cases}\frac{1}{70}(x-200), & \text { if } 200<x \leq 270 \\ 1, & \text { if } 270<x \leq 300 \\ 0, & \text { otherwise. }\end{cases}
$$

A simple calculation shows that $\mu_{A_{5}} \cap \mu_{A_{6}}=243.07$, which is the stationary point of the system, as shown in Proposition 15. This is the same result as that obtained from numerical experiments in Figure 13, where it is possible to observe the solution of the p-fuzzy system with initial condition $x_{o}=50$ converging to the stationary point $x^{*}=$ 243.07 (dotted line curve).

5.2. Example 2 (Application). Losses caused by fungi attacks reach up to the amazing and worrisome figure of $20 \%$ of the total harvested fruits. Among the recognized pathogens 


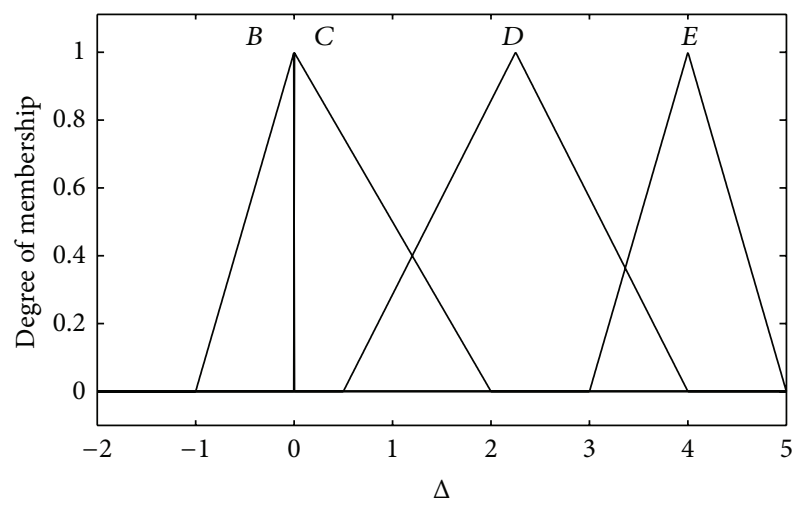

Figure 13: Equilibrium of the p-fuzzy systems.

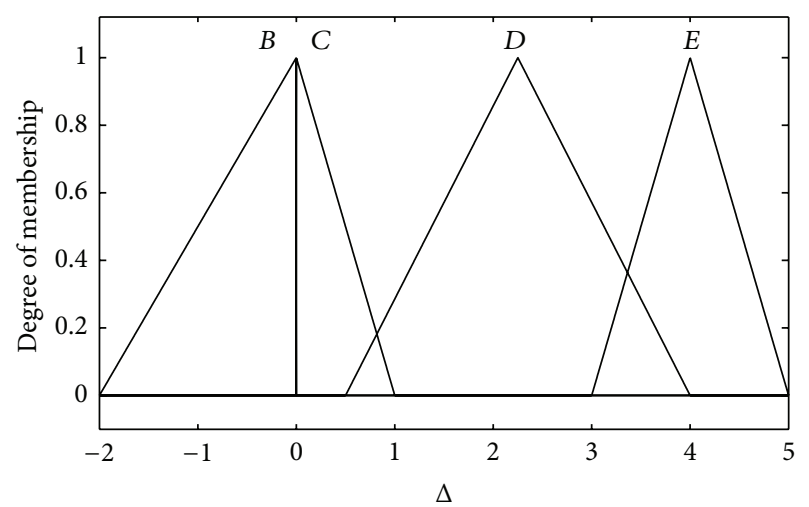

Figure 14: Population $(A)$.

of these degenerative processes are the Colletotrichum $s p$. (bitter putrefaction); Alternaria sp and Fusarium sp (carpelar putrefaction); Alternaria alternata (black putrefaction) and Penicillium sp. (blue mould). In general, putrefaction occurs always after harvesting, when the apples are kept in boxes awaiting the refrigeration process.

Let us consider a situation where an apple box filled with approximately 3000 fruits exists and there is a rotten apple in the center of the box that will contaminate the other apples. The dispersion of the disease occurs through the contact of the rotten apple with a healthy one. If we want to model the dispersion of the disease, we can only use the intuition because the available information we have is that after $n$ days all the apples will be contaminated. We do not have in hand a "force of infection of the disease" parameter, and we know little about the possible contacts between the rotten apple and a healthy one. Thus, any mathematical model or simulation of the phenomenon only will produce an approximate result. On the other hand, if we simply use the intuition, we can formulate some relative rules to the dispersion process such as the following:

"If the rotten apple population is "low" then the variation (incidence) of rotten apples is "small"."

Let us consider then the p-fuzzy system with the following linguistic variables:

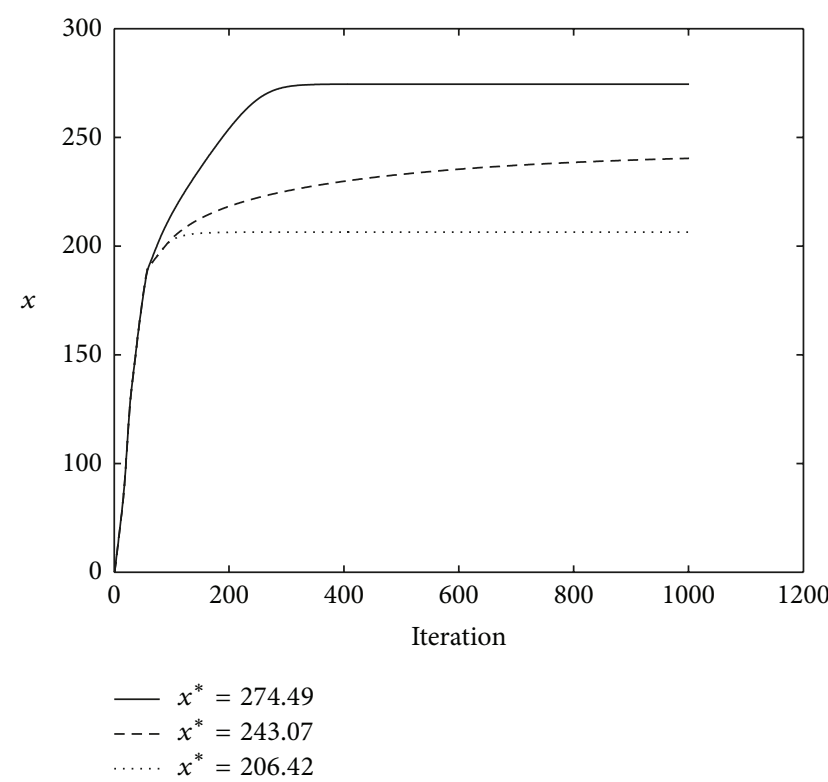

Figure 15: Variation $(\Delta)$.

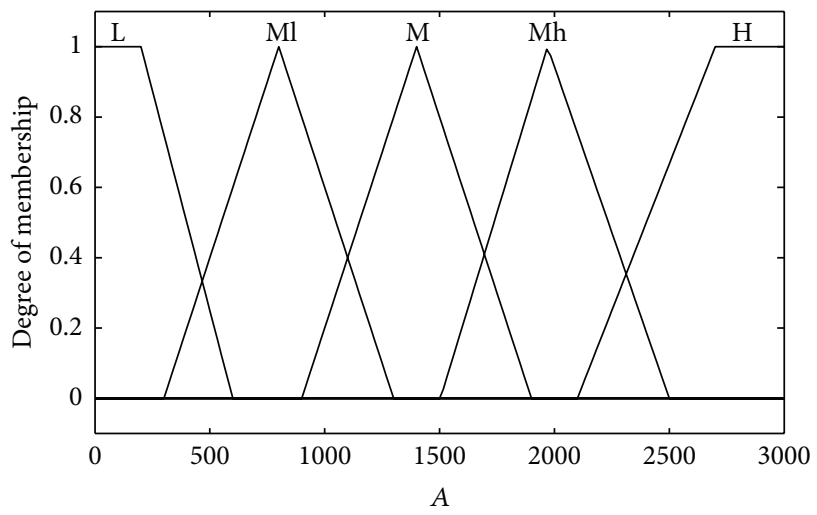

FIGURE 16: Solutions: p-fuzzy system and adjust model.

(i) A: "rotten apple population" (Figure 14); L: low; Ml: medium low; $\mathrm{M}$ : medium; medium high; $\mathrm{H}$ : high,

(ii) $\Delta$ "Variation" (incidence of the disease, Figure 15); S: small; M: medium; G: great,

where the rule-base is:

(1) if Population $(A)$ is low $(\mathrm{L})$, then Variation is small (S);

(2) if Population $(A)$ is medium low $(\mathrm{L})$, then Variation is medium $(\mathrm{M})$;

(3) if Population $(A)$ is medium $(\mathrm{M})$, then Variation is great $(\mathrm{G})$;

(4) if Population $(A)$ is medium high $(\mathrm{M})$, then Variation is medium $(\mathrm{M})$;

(5) if Population $(A)$ is medium $(G)$, then Variation is small (S). 
This p-fuzzy system can be used to adjust the parameters of a deterministic differential equation model of the type

$$
\frac{d A}{d t}=r A\left(1-\frac{A}{K}\right)
$$

for example, by the method of least squares. In Figure 16 it is possible to observe the solution of the p-fuzzy system (dotted line curve) and the adjust model (continuous line curve).

\section{Acknowledgment}

The authors would like to thank CAPES for the financial support.

\section{References}

[1] R. Fullér, Neural Fuzzy Systems, Abo Akademi University, 1995.

[2] T. Takagi and M. Sugeno, "Fuzzy identification of systems and its applications to modeling and control," IEEE Transactions on Systems, Man and Cybernetics, vol. 15, no. 1, pp. 116-132, 1985.

[3] F. Cuesta, F. Gordillo, J. Aracil, and A. Ollero, "Stability analysis of nonlinear multivariable Takagi-Sugeno fuzzy control systems," IEEE Transactions on Fuzzy Systems, vol. 7, no. 5, pp. 508-520, 1999.

[4] G. Feng, "Stability analysis of discrete-time fuzzy dynamic systems based on piecewise lyapunov functions," IEEE Transactions on Fuzzy Systems, vol. 12, no. 1, pp. 22-28, 2004.

[5] M. M. Gupta, "Energetic stability of dynamic systems," IEEE Transactions on Systems, Man, and Cybernetics B, vol. 15, no. 6, 1985.

[6] M. Johansson, A. Rantzer, and K.-E. Årzén, "Piecewise quadratic stability of fuzzy systems,' IEEE Transactions on Fuzzy Systems, vol. 7, no. 6, pp. 713-722, 1999.

[7] K. Tanaka, M. F. Griffin, and H. O. Wang, "An approach to fuzzy control of nonlinear systems: stability and design issues," IEEE Transactions on Fuzzy Systems, vol. 4, no. 1, pp. 14-23, 1996.

[8] K. Tanaka and M. Sano, "Concept of stability margin for fuzzy systems and design of robust fuzzy controllers," in Proceedings of the 2nd IEEE International Conference on Fuzzy Systems, pp. 29-34, San Francisco, Calif, USA, April 1993.

[9] H. Ying, "Structure and stability analysis of general mamdani fuzzy dynamic models," International Journal of Intelligent Systems, vol. 20, no. 1, pp. 103-125, 2005.

[10] M. Sugeno and T. Taniguchi, "On Improvement of stability conditions for continuous mamdani-like fuzzy systems," IEEE Transactions on Systems, Man, and Cybernetics B, vol. 34, no. 1, pp. 120-131, 2004.

[11] M. T. Misukoshi, Estabilidade de sistemas dinamicos fuzzy [Tese de Doutorado], IMECC-Unicamp, Campinas-SP, 2004.

[12] H. T. Nguyen and E. A. Walker, A First Course in Fuzzy Logic, Chapman \& Hall/CRC, Boca Raton, Fla, USA, 3rd edition, 2006. 


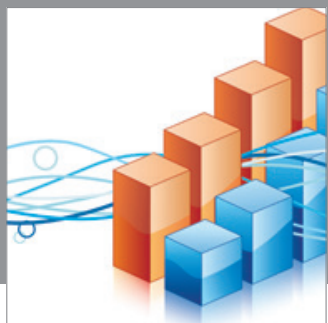

Advances in

Operations Research

mansans

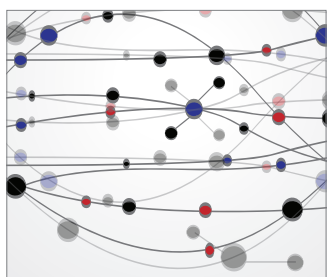

The Scientific World Journal
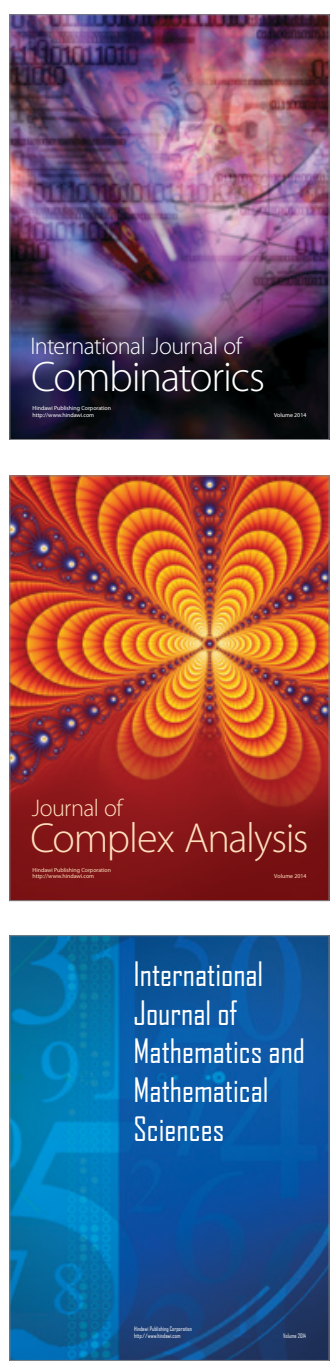
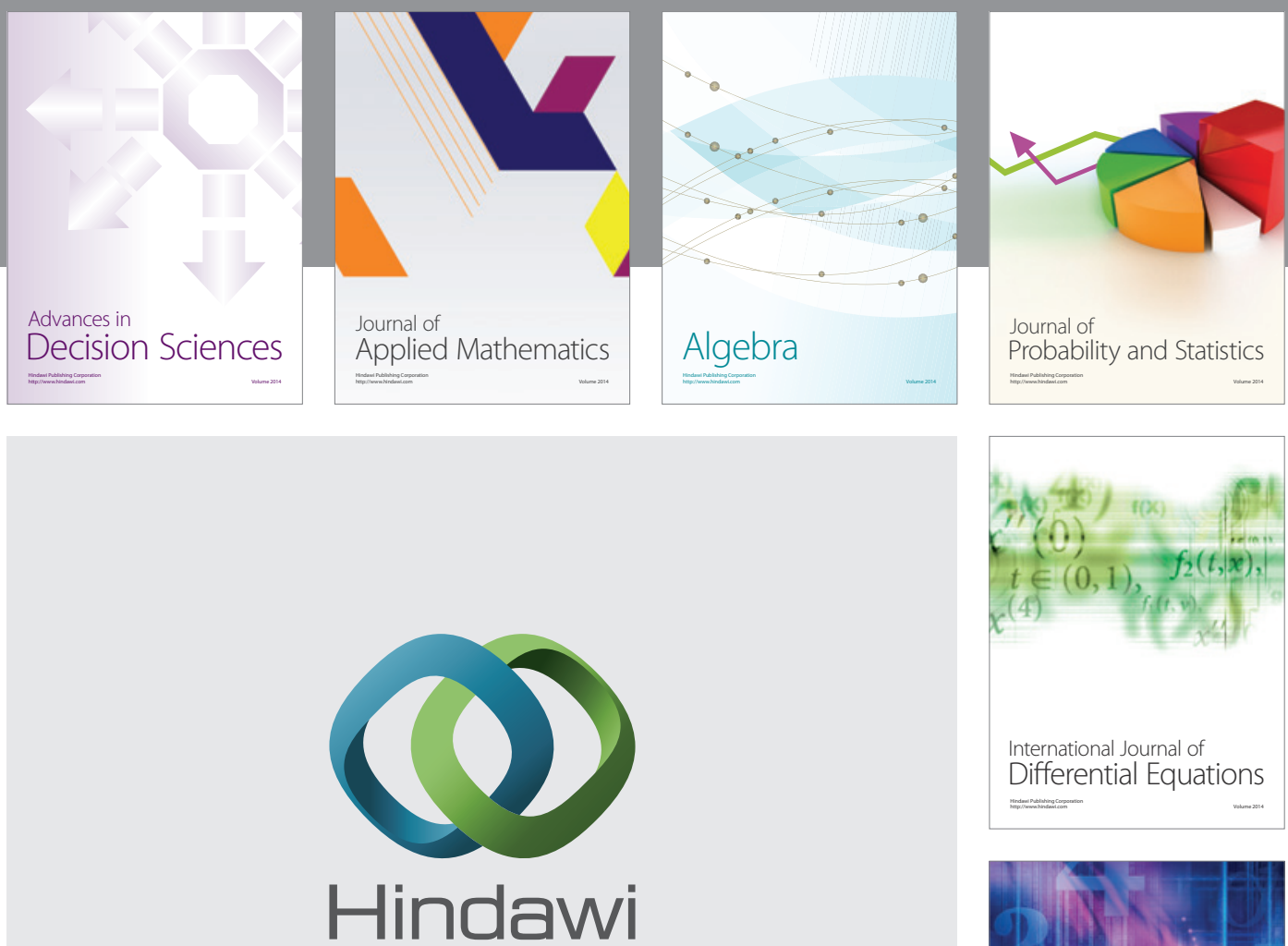

Submit your manuscripts at http://www.hindawi.com
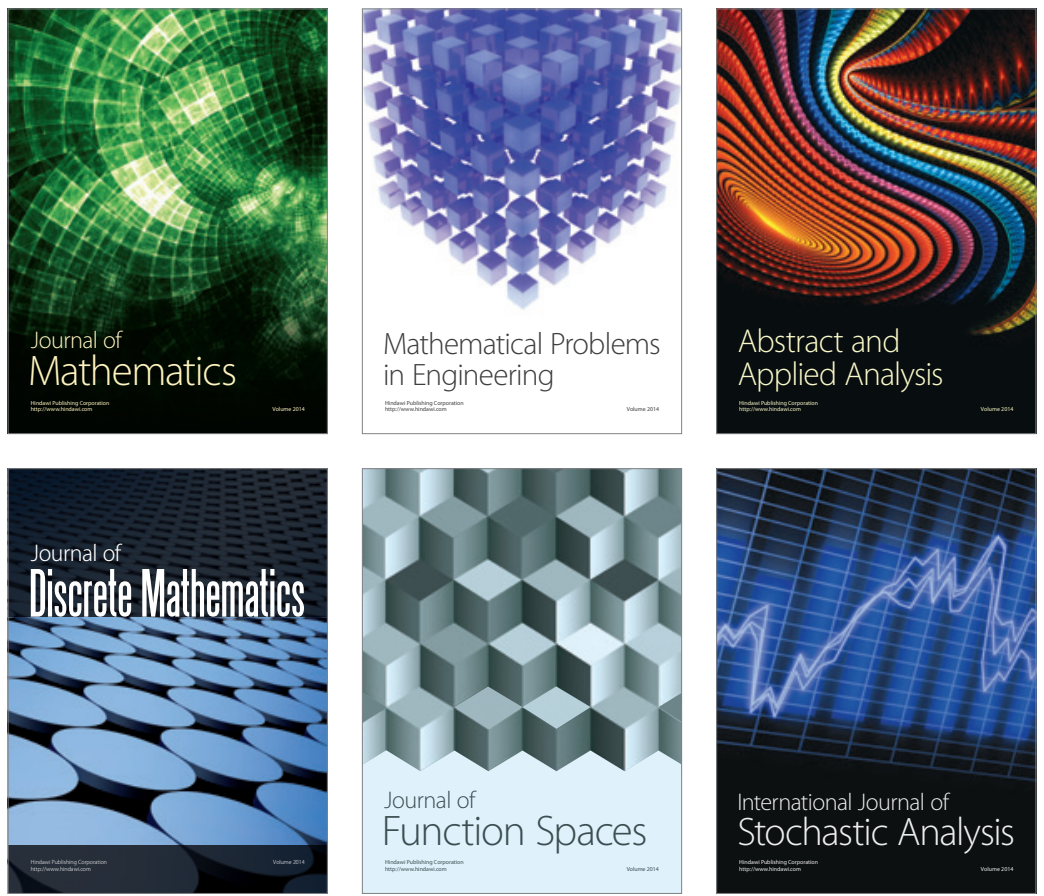

Journal of

Function Spaces

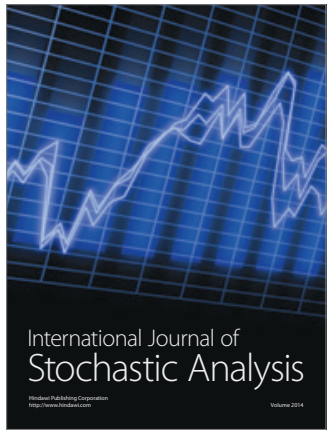

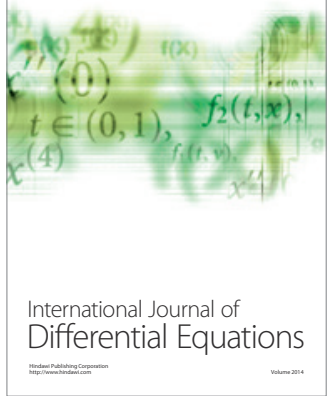
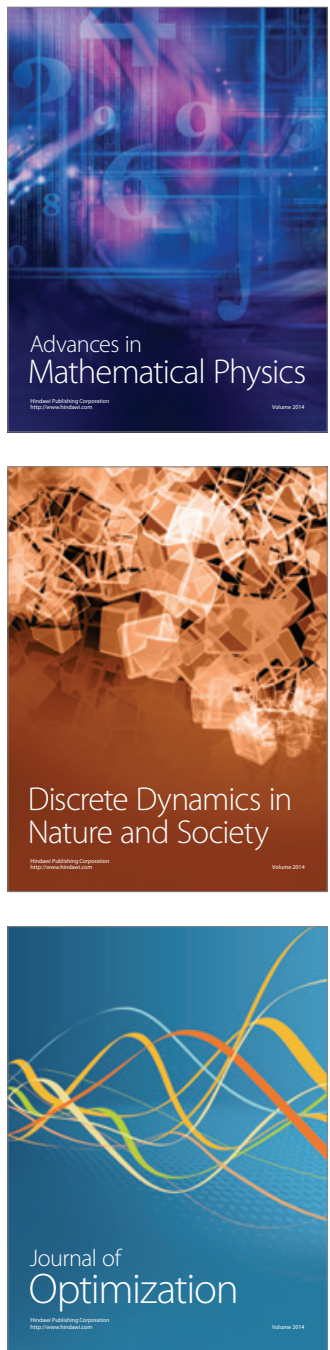